Revue d'histoire de l'Amérique française

REVUE D.HISTOIRE DE L'AMÉRIQUE FRANÇAISE

\title{
Il y a cent ans : le démembrement de la paroisse Notre-Dame
}

\section{Léon Pouliot}

Volume 19, numéro 3, décembre 1965

URI : https://id.erudit.org/iderudit/302490ar

DOI : https://doi.org/10.7202/302490ar

Aller au sommaire du numéro

Éditeur(s)

Institut d'histoire de l'Amérique française

ISSN

0035-2357 (imprimé)

1492-1383 (numérique)

Découvrir la revue

Citer cet article

Pouliot, L. (1965). Il y a cent ans : le démembrement de la paroisse Notre-Dame. Revue d'histoire de l'Amérique française, 19(3), 350-383.

https://doi.org/10.7202/302490ar d'utilisation que vous pouvez consulter en ligne.

https://apropos.erudit.org/fr/usagers/politique-dutilisation/ 


\section{IL Y A CENT ANS : LE DEMEMBREMENT DE LA PAROISSE NOTRE-DAME}

Le 22 décembre 1865, la Congrégation de la Propagande approuvait un décret relatif au démembrement de la paroisse de Ville-Marie, et dont les effets heureux se sont prolongés jusqu'à nous. Il avait pour but de mettre fin au malaise, pour ne pas dire à l'antagonisme, qui existait entre le Séminaire et l'évêché, et du même coup, assurer un service pastoral mieux adapté aux besoins nouveaux d'une population qui s'accroissait rapidement. Dans l'immédiat, le décret fut loin de produire tout le bien qu'on en avait espéré. Son exécution provoqua même un autre combat qui, par sa durée et son intensité, ne laisse pas de nous étonner aujoưrảhüi: elle noüs valüt aüssi toute une littérature passionnée, plus défensive et agressive qu'historique, et où il n'est pas toujours facile de voir clair.

Nous tenterons ici de raconter les circonstances qui ont préparé immédiatement le décret de 1865 . C'est dire que nous laissons délibérément de côté tous les faits et événements qui lui sont postérieurs. Quant à l'opposition qui avait existé jusque-là entre le Séminaire et l'évêché, nous n'en retenons que ce qui est indispensable au sujet. Prenant les choses où elles en étaient en l'an de grâce 1864, nous nous proposons de décrire la laborieuse naissance du décret de 1865. Entrons en matière.

I

\section{LA PAROISSE DE MONTREAL EN 1864}

Le 9 novembre 1864, Mgr Bourget rédigeait, à l'adresse du cardinal Barnabo, Préfet de la Propagande, un long mémoire.

On y lisait ceci:

La cure de Montréal, qui comprend la ville et la banlieue, forme une immense paroisse de 11 milles dans 
sa plus grande longueur, sur $51 / 3$ milles dans sa plus grande largeur. Le nombre des fidèles qui composent le peuple de l'église paroissiale s'est élevé dans les trois dernières années de 75,000 à 80,000 âmes. Telle est la paroisse qui se trouve encore aujourd'hui, comme elle le fut dès son origine, unie et annexée au Séminaire de St-Sulpice, et à la tête de laquelle se trouve le Supérieur de la Maison, qui en devient le curé par le fait de son élection au supériorat de la communauté. ${ }^{1}$

Nous avons ici les deux éléments essentiels du problème. Le premier est d'ordre pastoral: c'est l'immensité de la paroisse tant par son étendue que par le nombre de ses fidèles. Mgr Bourget ne pouvait y rester indifférent. Comme premier Pasteur du diocèse, il avait charge d'âmes, de toutes les âmes qui vivaient dans le territoire de sa juridiction. Or, partout ailleurs, il pouvait s'acquitter de ce devoir, soit par le jeu de la nomination aux cures, soit par la division des paroisses devenues trop nombreuses. Mais, à Montréal, il n'avait pas ses coudées franches.

Le second élément du problème était d'ordre canonique. Ordinaire du lieu, Mgr Bourget était le gardien de la discipline ecclésiastique. Or, d'après celle-ci, toute nomination ad curam animarum, et surtout celle des curés, vient de l'évêque et toute paroisse reste en droit et en fait, sous la pleine et entière dépendance de l'Ordinaire. Mais dans la Paroisse, il n'en allait pas de même. Le Supérieur était élu tous les cinq ans par le Conseil du Séminaire: élection qui se déroulait selon les constitutions de Saint-Sulpice, mais à laquelle l'évêque était complètement étranger: une fois élu, il devenait automatiquement curé de VilleMarie, et il déléguait ses inférieurs ad curam animarum sans aucune participation de l'évêque.

De temps en temps, pour l'acquit de sa conscience, pour empêcher la proscription de ses droits, Mgr Bourget les affirmait. Ainsi, en 1843, au moment même où il confirmait et ratifiait les privilèges du Séminaire, il investissait publiquement curé de Ville-Marie, M. Joseph-Vincent Quiblier qui, Supérieur du Sémi-

${ }^{1}$ ACM. Lettres de Mgr Bourget, 14: 162. 
naire, en exerçait les fonctions depuis 12 ans déjà. ${ }^{2}$ Et vingt ans plus tard, il adressait au Supérieur, M. Dominique Granet, des lettres de chapelain pour le confesseur de la Congrégation:

Ce n'est toutefois que par hasard que j'ai appris la nomination de M. Lacan, comme j'apprends d'ordinaire les changements que vous faites chaque année. Assez souvent, c'est le calendrier, quand il est imprimé, qui me les fait connaître. Il arrive de là que je ne sais à qui m'adresser, quand il faut correspondre avec les missionnaires du Lac et des faubourgs, ou avec les chapelains des communautés.

Ce n'est que par hasard que j'ai appris que $\mathrm{Mr}$. Sentenne avait remplacé Mr Deguire à l'Hotel-Dieu. S'il y a eu d'autres changements, je n'en sais rien. Il m'est très pénible d'en venir encore à de telles explications, et je ne puis m'expliquer cette extrême réserve, qui me fait croire que l'on craint l'action de l'évếque dans la direction de la paroisse, qu'il a confiée aux soins du Séminaire.

Je n'en suis pas moins disposé à vous seconder dans toutes vos bonnes et saintes œuvres, et suis et serai toujours, de vous Mr le Supérieur, et de tous vos Messieurs, le très dévoué serviteur. ${ }^{3}$

La réserve dont s'étonne Mgr Bourget a pourtant son explication. Si le Séminaire fait la sourde oreille, c'est qu'il se croit dans son bon droit: il se regarde comme investi directement par le Saint-Siège de la desserte de la Paroisse, de l'unique paroisse de Montréal. Sur quoi se base-t-il ?

II

\section{LES PRIVILEGGES DU SEMINAIRE DE MONTREAL}

A notre connaissance, les droits et privilèges du Séminaire de Montréal sont contenus dans les trois documents suivants:

2 ACM. Paroisse Notre-Dame, 1678-1865. No 6. Copie du mandement de l'évêque de Montréal concernant le curé de Notre-Dame de Montréal, 24 mai 1843.

3 ACM. Lettres de Mgr Bourget, 13: 46-47, 4 septembre 1863. 
1 - Acte de Mgr de Laval (1678), érigeant canoniquement la paroisse de Ville-Marie et l'unissant pour toujours au Séminaire de Montréal. ${ }^{4}$

2-Ordonnance de Mgr de Saint-Vallier (1694), décrétant que le Supérieur du Séminaire sera à perpétuité curé de Ville-Marie. ${ }^{5}$

3 - Lettre du cardinal Pedicini (1831), Préfet de la Propagande, à l'occasion d'un différend survenu entre l'évêque de Québec, Mgr BernardClaude Panet et le Séminaire de Montréal. ${ }^{6}$

Si l'on s'en tient au droit écrit, il faut reconnaître que les ordonnances de Mgr de Laval et de Mgr de Saint-Vallier avaient réservé les droits de l'Ordinaire sur la paroisse et qu'elles ne liaient en aucune façon ni leurs successeurs sur le siège de Québec, ni, à plus forte raison, l'évêque de Montréal à partir de 1836, date de l'érection du diocèse. Mgr Bourget écrivait avec raison en 1864 :

Ce qu'il faut conclure des deux ordonnances qui ont réglé la cure de la paroisse de Montréal et des changements apportés aux constitutions du Séminaire de cette ville, c'est que cette communauté a, comme toutes les autres, le droit d'élire son supérieur, mais aussi comme toutes les autres, le devoir de le présenter à l'ordinaire pour qu'il soit nommé curé actuel de la paroisse unie, dont le Séminaire est le curé habituel. Mais on ne peut nullement en conclure que ce dernier puisse installer son supérieur ou le curé élu, sans qu'il soit présenté à l'évêque pour être par lui approuvé comme tel... ad curam animarum.7

4 ACM. Paroisse Notre-Dame, 1678-1865, no 1. Texte du document par lequel Mgr de Laval érige Ville-Marie en paroisse, sous le titre de la Bienheureuse Vierge Marie et nomme S. Sulpice cure in perpetuum. 30 novembre 1678.

5 Ibid., no 2 - Texte par lequel Mgr de Saint-Vallier unit les cures de l'Ile de Montréal au Séminaire de Saint-Sulpice 3 août 1694. Note d'archive de la main de Mgr Bourget: "Mandement de Mgr de Saint-Vallier établissant le supérieur du Sém. de Montréal, curé de Ville-Marie."

6 Ibid., no 5 - Note d'archive de Mgr Bourget: "Décision du card. Préf. de la Prop. concernant la cure de N.-D. de Montréal, 19 décembre 1831, card. Pedicini, Préf."

7 ACM, Lettre déjà citée de Mgr Bourget au cardinal Barnabo. 9 novembre 1864 . 
C'était pourtant cette exemption de la juridiction de l'Ordinaire, dont il se croyait en légitime possession, que le Séminaire demandait au Saint-Siège de confirmer. Son grand argument: son cheval de bataille ? La lettre du cardinal Pedicini, en date du 19 décembre 1831. Force nous est d'en raconter ici les circonstances et d'en dire le sens véritable.

Les circonstances: Si les textes écrits maintenaient les droits de l'Ordinaire sur la Paroisse, un usage s'était établi qui, en se perpétuant, allait jouer en faveur du Séminaire. Insensiblement, les évêques de Québec se désintéressèrent de Montréal: à partir de 1787, ils n'y font même plus la visite canonique ${ }^{8}$ pourtant prescrite par les saints canons. D'autre part, conséquence de son zèle, du cœur qu'il mettait à la besogne, des sacrifices financiers qu'il consentait généreusement pour le progrès de la Paroisse, le Séminaire en vint à considérer celle-ci comme sa chose.

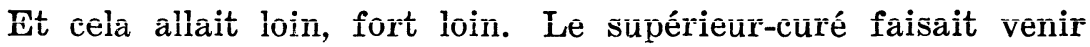
des Sulpiciens de France sans en avoir obtenu la permission de l'évêque: et sous prétexte qu'il avait assez de sujets, il refusait de recevoir au Séminaire des prêtres du pays: ${ }^{9}$ il donnait luimême à ses inférieurs la délégation ad curam animarum et il se déchargeait sur un confrère des fonctions curiales sans présenter celui-ci à l'approbation de l'évêque. ${ }^{10}$ En 1808, Mgr Plessis avait voulu fixer à Montréal la résidence de son coadju-

8 AAM, Pièces et Actes, IV: 189v-190. Ordonnance de visite pour la paroisse de Ville-Marie, en l'Isle de Montréal (12 mars 1843). Mgr Bourget y note que la dernière visite épiscopale remonte à 1787 et qu'elle fut faite par Mgr Pierre Denaut.

9 ACM, Québec, 1830-1831. Mgr Panet à M. Roux, supérieur du séminaire 12 juillet 1830: "C'est avec la plus grande surprise que j'ai été informé que vous paraissiez vouloir refuser d'admettre dans votre maison deux prêtres canadiens d'un caractère irréprochable (...) Mais ce qui me surprend encore davantage, c'est que le refus que vous faites d'admettre ces deux Mrs au Séminaire de S. Sulpice, ne paraît motivé que par le prétexte que vous attendez de nouveaux prêtres français. Il n'y a donc plus à douter que votre intention ne soit de composer cette maison de prêtres étrangers, au préjudice et à l'exclusion de ceux du pays." MM. Brien et Girouard s'étaient vu refuser l'entrée au séminaire. Pourquoi ? On ne le saura jamais; car les Sulpiciens ont rejeté l'accusation portée ici contre eux. Malgré cela, l'attitude du Séminaire fut interprétée par Mgr Panet et par son informateur Mgr Lartigue comme dictée par le nationalisme français. Et le fait du refus contribua à rendre plus tendues les relations entre le Séminaire et l'évêque.

10 On appelait ce personnage le curé d'office. 
teur; M. Roux refusa de le recevoir au Séminaire, et les choses en restèrent là: il n'y avait de place à Montréal, semble-t-il, que pour des prêtres sulpiciens. ${ }^{11}$

En 1821, le Séminaire tenta de faire obstacle à la résidence en ville de Mgr Jean-Jacques Lartigue, évêque de Telmesse, et préposé par le Saint-Siège à l'administration spirituelle du District de Montréal. ${ }^{12}$ Cette fois, il avait trop présumé de ses forces. Son opposition allait accélérer la construction, au cœur même de la Paroisse, d'une église non sulpicienne, Saint-Jacques, comme nous l'apprend Mgr Plessis lui-même:

Dieu, qui sait tirer sa gloire de tout, Nos très chers frères, a pris occasion des contrariétés par lesquelles Mgr l'Evêque de Telmesse a été éprouvé depuis le commencement de son Episcopat, pour procurer à votre ville une église de plus, dont la magnifique structure s'est élevée comme par enchantement et est parvenue à sa fin avec une rapidité dont les étrangers et les indigènes sont également surpris. Nous en félicitons tous ceux qui y ont contribué de leurs soins ou de leurs aumônes, tous ceux qui assisteront à la consécration qui s'en fera prochainement, comme Nous Nous proposons d'y assister Nous-mêmes, tous ceux qui auront par la suite la consolation d'y adorer Jésus-Christ, d'y assister aux Saints-Offices, d'y entendre la parole de Dieu, d'y déposer leurs péchés et d'y recevoir les sacrements. ${ }^{13}$

Cet enracinement de Mgr Lartigue dans la Paroisse n'avait fait qu'accentuer l'opposition entre le Séminaire de Montréal

11 Prop. Scrit. rifer., 1792-1830. II : 370 et ss. Mgr Plessis à M. Robert Gradwell, 17 décembre 1822. - Mgr Panet aurait forcément habité au séminaire et officié à Notre-Dame aux jours de fête, puisqu'il n'y avait alors en ville aucune autre résidence presbytérale ni aucune église digne d'un évêque. On a soutenu parfois que M. Roux s'opposait non à la présence de l'évêque coadjuteur dans la ville, mais à sa résidence au Séminaire, parce qu'il la croyait incompatible avec les privilèges et la liberté de celui-ci. Si tel était le cas, on comprendrait que le séminaire ait mal accueilli la présence de Mgr Lartigue au séminaire, après son sacre en 1821 , et qu'il ait souhaité le voir s'installer dans une paroisse rurale. Mais on comprend bien que le Séminaire ait continué son opposition à Mgr Lartigue quand, à partir de 1825, celui-ci a sa résidence et son église dans la ville.

12 Sur ce sujet, voir notre étude Mgr Bourget et son Temps, I: 53 ss. 13 RAPQ, (1932-1933) : 237. 
et l'évêque de Québec. Celui-là affichait toujours envers celui-ci une indépendance qui paraissait peu compatible avec la discipline de l'Église. Pour des raisons qu'il n'est pas nécessaire de détailler ici, en 1830, Mgr Panet, archevêque de Québec, voulut exercer les droits qu'il possédait sur la Paroisse comme sur toutes les paroisses du diocèse. Depuis plus de cent ans, c'était la première fois que l'Ordinaire de Québec parlait si haut. Le Supérieur du Séminaire déféra le cas à Rome et Mgr Panet en fit autant. ${ }^{14}$

L'assemblée générale de la Propagande fut appelée à se prononcer le 19 septembre 1831. Après avoir mûrement pesé les arguments mis en avant par les deux parties en présence, elle manifesta sa pensée en quatre points: $1^{\circ}$ ) dans une question de cette nature, le fait des évêques prédécesseurs ne diminue ou ne lie en rien l'autorité des successeurs. C'était dire qu'en toute rigueur de droit, Mgr Panet était libre de mettre fin aux privilèges accordés par ses prédécesseurs au Séminaire de Montréal: $2^{\circ}$ ) relativement à la nomination par le Supérieur du curé d'office, les cardinaux émettaient l'avis que l'assentiment ininterrompu des évêques de Québec pendant un si long espace de temps (137 ans) méritait des égards: et cela signifiait que, in concretis, il n'était pas facile d'abroger cet usage purement et simplement: $3^{\circ}$ ) ils étaient d'avis que la controverse prît fin par une entente cordiale (amichevole concordia) entre les parties : $4^{\circ}$ ) et que, pour obtenir cette fin, des lettres seraient adressées en ce sens aux deux parties, au nom de Sa Sainteté. "Nous désirons que les deux parties s'entendent, concluait le procès-

14 Il s'agit de la nomination de M. John Jackson Richards, comme curé d'office. Mgr Panet écrit à M. Roux, le 12 juillet 1830: "Pour vous donner lieu de reconnaître combien j'étais disposé à vous satisfaire en toute manière possible dans l'élection du curé d'office, je vous avais prié de me communiquer les titres en vertu desquels vous vous croyez autorisé en qualité de supérieur du Séminaire de choisir pour l'exercice des fonctions curiales celui des membres de votre maison qu'il vous plaira, sans être obligé ensuite de le présenter à l'approbation de l'évêque. Vous n'avez pas jugé à propos de me communiquer ces titres, mais constant dans votre droit exclusif de faire la dite élection sans l'approbation de l'Ordinaire, vous m'avez signifié votre disposition de remettre cette affaire à la décision de la Cour de Rome. C'est à quoi je me suis déterminé de mon côté, et je me propose d'écrire prochainement à cette Cour en conséquence." $A C M$., Québec, $1830-31$. 
verbal de la réunion; et c'est pourquoi, qu'on n'innove pas, et dans la mesure où la chose serait nécessaire, il faudrait demander au Saint-Père de valider les actes du passé. ${ }^{15}$ L'essentiel est là : pour le passé, validation par le Saint-Père des irrégularités, s'il y en a eu: pour le présent, pas de changement: pour l'avenir une entente à conclure entre les parties. Il appartenait au cardinal Pedicini, préfet de la Propagande, d'exécuter la pensée des cardinaux en écrivant à Mgr Panet et au Séminaire de Montréal. Il s'acquitte de ce devoir le 19 décembre 1831.

Sa lettre à Mgr Panet s'inspire du procès-verbal, mais elle le dépasse parfois: de plus elle manque de clarté et de sérénité. Elle apparaît plus que celui-ci favorable au but que poursuit le Séminaire. Ainsi, le Placere de concordia... que nous avons cité plus haut devient: "ils (les cardinaux) ont décidé que la controverse doit finir par une entente mutuelle, qui aura pour effet de maintenir les choses dans l'état où elles ont été jusqu'ici." 16 Interprétation qui rendait en quelque sorte obligatoire et perpétuel le nihil innovetur du procès-verbal. Or, l'expression avait, croyons-nous, un sens beaucoup plus restreint. En effet, eu égard au fait que les erreurs possibles du passé sont corrigées par le Souverain Pontife et au fait que l'avenir sera assuré par une entente entre les parties, il est tout naturel de penser que le nihil innovetur s'entend du moment présent: aucune des parties ne doit prendre sur elle de régler unilatéralement la question. En d'autres termes, le curé d'office dont le droit est contesté par l'évêque reste au poste en attendant la solution finale.

De plus, la lettre du cardinal Pedicini semble attribuer à une initiative du Pape le souci qu'il a de vouloir rectifier les erreurs possibles du passé. ${ }^{17}$ Or, d'après le procès-verbal, ce

15 Prop. Acta 1831, 401 et ss. "Placere de concordia; et idcirco nihil innovetur, et quatenus opus sit, supplicandum Smo pro sanatione."

16 Les cardinaux: "statuerunt controversiam finiri opportere per mutuam concordiam, quæ fiat ut res circa negotium hoc in eo statu relinquantur in quo hactenus fuerunt."

17 "SSmo dno no Gregorio PP. XVI, S.C. responsio relata est. S.S. vero non modo illam probavit, sed et jubere dignata est A.T. nomine suo eamdem responsionem significari, addita sanctione Pontificiæ pro casu quo necessaria existimetur ad confirmandum Tuorum Præderessarum factum super materia de qua agitur." 
geste lui a été demandé par les cardinaux. Le Pape approuve le contenu du procès-verbal; il ne va pas plus loin: pour lui comme pour les cardinaux, l'entente à venir est actuellement entre les mains des parties intéressées. Or, ce sont ces deux malheureuses phrases, qui ont permis de prêter à la lettre du cardinal Pedicini une autorité souveraine qu'elle n'avait pas. Ce n'est pas tout. Dans l'énervement du moment, on n'a tenu aucun compte de la dernière phrase dans laquelle, le cardinal, se reposant sur l'obéissance de Mgr Panet au Saint-Siège et sur son respect pour la Propagande, se déclare "certain que les deux parties en viendront sans retard à une entente, entente qui durera à jamais". ${ }^{18}$

Résumons-nous: inviter les parties à s'entendre, tel était le mandat que le cardinal Pedicini avait reçu de l'assemblée générale de la Propagande: tel était aussi ce à quoi Grégoire XVI pensait, quand il donnait son approbation. Dans la mesure où la lettre du cardinal Pedicini introduisait des éléments nouveaux que ne contenait pas le procès-verbal de l'assemblée générale, elle est la manifestation de sa pensée personnelle: elle n'est autorisée ni par la Propagande ni par l'approbation de Grégoire XVI.

Tel est le document que le Séminaire considérait comme la grande charte de son indépendance envers l'Ordinaire du lieu. Dans le mémoire que MM. Carrière, supérieur général et Baile, délégué de Montréal, présentent au cardinal Barnabo en 1863,19 la lettre du cardinal Pedicini est qualifiée de décision de la Propagande; ils en demandent la ratification solennelle comme moyen de ramener entre le Séminaire et l'évêché cette paix et ce bon ordre que l'un et l'autre désirent ardemment.

Quant à Mgr Bourget, il ne voyait de paix que dans la juste dépendance du Séminaire à l'évêque. A ses yeux, ce problème était le plus important, le plus urgent; et il contenait en germe la solution du problème pastoral. A Rome, où il y avait 60 parois-

18 "ut certus sim concordiam continuo initum iri et eam perpetuo esse duraturam."

19 ACM, Paroisse Notre-Dame, 1678-1865, no 12. 
ses, le cardinal Barnabo avait été étonné d'apprendre en 1855, qu'il n'y en avait qu'une à Montréal, et il avait suggéré à Mgr Bourget d'exposer la situation à la Propagande. Celui-ci s'y était refusé, tant il avait horreur d'étaler aux yeux du Saint-Siège les difficultés qu'il avait avec son clergé. Il comptait sur le temps pour apporter au problème une solution pacifique.

Le Séminaire souffrait également du malaise qui régnait à Montréal. Il avait l'impression que l'évêque ne lui était pas sympathique, et il n'arrivait pas à en comprendre le pourquoi. Il se disait prêt, et il était sincère, à exaucer les désirs de l'évêque dans la mesure où il le pourrait financièrement et dans la mesure où son indépendance, garantie par la lettre du cardinal Pedicini, ne serait pas compromise. Or, ces restrictions ou conditions s'accordaient mal avec la conception que Mgr Bourget avait de ses droits et devoirs comme évêque de Montréal. De deux choses l'une, pensait-il. Ou bien la lettre du cardinal avait, en 1831 , le caractère impératif qu'on lui a attribué. Mais elle est dépassée par les événements, par le rapide accroissement de la population et par la distribution de celle-ci sur un vaste territoire. Un privilège n'a plus sa raison d'être quand il devient plus nuisible qu'utile au bien des âmes. Ou bien, la lettre du cardinal n'était pas impérative en 1831 , et elle ne l'est pas davantage aujourd'hui.

Dans un cas comme dans l'autre, on s'appuyait sur Rome pour le limiter dans l'exercice de ses droits et devoirs. Dès lors, pensait-il, c'est à Rome de parler, de déterminer le sens du document et, si elle le juge à propos, de le déclarer désormais nul.

En fait, c'est le Séminaire qui le premier eut recours au Saint-Siège. En 1860, le Supérieur, M. Dominique Granet, se rend à Rome. Le cardinal Barnabo lui prêta une oreille attentive et prit sur lui de régler le problème. On n'explique pas autrement cette demande de Mgr Bourget au cardinal préfet, à la date du 8 décembre 1862:

Un décret apostolique pour régler canoniquement la desserte de la ville et paroisse de Montréal par les Ecclésiastiques de St-Sulpice qui désirent con- 
server l'intégrité de cette paroisse, qui a cent mille âmes, en faisant des résidences quasi-curiales, après que Son Eminence le cardinal Préfet de la S. Cong. de la Prop. aura fixé, comme elle a bien voulu se charger de le faire, les droits de l'Evêque et les privilèges dont pourront jouir les dits Ecclésiastiques comme curés de cette grande paroisse, divisée en quartiers pour y faire facilement les fonctions curiales. Cette faveur est sollicitée pour terminer tous les différends qui pourraient troubler la bonne harmonie entre les divers ouvriers qui travaillent dans le même champ du Père de famille. ${ }^{20}$

Le cardinal demande alors au Séminaire de lui faire connaître son point de vue et de lui manifester ses désirs. Muni d'une lettre de recommandation de Mgr Bourget, M. Joseph Baile, p.s.s. se dirige sur Paris, et de là accompagné du Supérieur général, M. Carrière, il se rend à Rome. Le mémoire qu'ils présentent au cardinal Barnabo, le 11 juillet $1863,{ }^{21}$ est une justification de l'attitude, des œuvres, de l'esprit du Séminaire. Celui-ci, affirmait le document, a été dès l'origine et est encore aujourd'hui rempli de respect et de soumission envers l'Ordinaire; il n'a rien négligé pour adapter le ministère pastoral aux besoins d'une population toujours grandissante, témoin les cinq ou six grandes églises qu'il a érigées et qu'il entretient à ses frais pour l'avantage des paroissiens dispersés sur un vaste territoire. ${ }^{22}$ Les prêtres du Séminaire sont dévoués, fervents, d'une vie exemplaire, comme Mgr Bourget a su lui-même le reconnaître. ${ }^{23} \mathrm{Et}$ pourtant, l'évêque de Montréal n'est pas content. Que veut-il ?

20 ACM, Lettres de Mgr Bourget, 12: 637-640.

21 ACM, Paroisse de Notre-Dame, 1678-1865, no 8.

22 Il s'agit croyons-nous des églises suivantes: Notre-Dame-de-Grâce (1850); Sainte-Anne (1854); Saint-Jacques (1857 et 1860); St-Joseph (1861-1862); Saint-Vincent-de-Paul (1858). A quoi il faudrait ajouter l'église Saint-Patrice, due à la générosité du séminaire et à l'esprit d'entreprise de M. Quiblier, son supérieur d'alors. Inaugurée le 17 mars 1847. Voir Gauthier, Sulpitiana, 260.

23 Dans le mandement de visite de la paroisse, qui est du 23 mai 1843, Mgr Bourget avait écrit: "Nous ... désirons donner une nouvelle preuve de la haute estime que nous portons à Messieurs les Ecclésiastiques du Séminaire de St-Sulpice pour les services qu'ils ont rendus à la religion et, en particulier pour avoir, de tout temps, gouverné cette paroisse avec tant de zèle et d'édification..." ACM, Paroisse de Notre-Dame, 1678-1865, no 6. 
On lui a demandé des précisions et il s'y est refusé. ${ }^{24}$ Il lui est arrivé de méconnaître les droits du Séminaire sans protestation de la part de celui-ci. ${ }^{25}$. Il n'est pas désirable qu'il intervienne dans les affaires de la Paroisse; car ce serait semer un germe de division dans une communauté de prêtres intimement unis entre eux et qui semble avoir reçu de Dieu une mission particulière pour Montréal. Que demandent MM. Carrière et Baile? Le Statu quo: intégrité de la paroisse, autorité exclusive du Supérieur sur tous les prêtres du Séminaire:

Votre Eminence le voit, nous ne demandons pas de choses nouvelles, nous sollicitons simplement le maintien de l'ordre établi; notre unique désir est de travailler de plus en plus avec zèle et générosité à cultiver cette partie de la vigne du Seigneur, qui paraît nous être échue en partage par un effet particulier de la volonté de Dieu, comme il paraîtra clairement par l'histoire de cette colonie, ${ }^{26}$ à laquelle travaille très activement l'auteur de la Vie de $M$. Olier et des Monuments inédits sur l'Apostolat de Ste-Magdeleine en Provence.

Nous serions au comble de nos vœux, si la S.C. de la Propagande, continuant de suivre la ligne bienveillante qu'elle a suivie jusqu'ici, daignait: $1^{\circ}$ ) confirmer la réponse déjà donnée en 1831, et déclarer qu'elle maintient le statu quo jusqu'à ce qu'il en soit ordonné autrement; $2^{\circ}$ ) faire connaître cette intention à Monseigneur de Montréal; $3^{\circ}$ ) l'engager, s'il a de nouveaux arrangements ou modifications à proposer, à les faire connaître d'abord aux MM. de S. Sulpice, afin que tout se règle pacifiquement, comme il en a témoigné souvent le désir, comme le désirent aussi la $\mathrm{S}$. Cong. et la compagnie de S. Sulpice: que si les deux parties ne peuvent s'accorder ensemble, l'affaire sera de nouveau dé-

24 Le silence de Mgr Bourget s'explique par le fait qu'à ses yeux le problème est sub judice. Comme nous l'avons signalé plus haut, le cardinal Barnabo s'en était réservé la solution en 1860 .

25 "Les droits du séminaire": tels que compris dans la lettre du cardinal Pedicini du 19 décembre 1831. Nous avons signalé qu'en effet Mgr Bourget contestait l'interprétation que le séminaire donnait à ce document. 26 L'histoire de la colonie française de M. Etienne-Michel Faillon, qui sera publiée en 1865-1866. 
férée au S. Siège, dont la décision nous trouvera toujours entièrement soumis.

Par son contenu, par l'attachement qu'il manifestait au Saint-Siège, par les ombres qu'il jetait sur la figure de Mgr Bourget, le Mémoire de MM. Carrière et Baile était important. Il posait le problème, il suggérait une solution, celle qui lui paraissait la meilleure.

Le cardinal Préfet se tourne alors vers Mgr Bourget; il lui transmet le Mémoire et il lui demande d'y répondre point par point. La réponse de l'évêque est du 29 septembre 1863. Document monumental par sa longueur: 43 pages grand format. Pour une meilleure intelligence de la pensée de Mgr Bourget, il ne sera pas inutile d'en citer le début et de noter la fin qu'il poursuit.

Dans sa lettre du 17 juillet dernier, que je reçus le 20 courant, Votre Eminence m'enjoint de répondre à tous les points du Mémoire de M. Baille concernant la cure de Montréal. J'obéis, mais avec une souveraine répugnance, parce que je ne puis le faire sans contrister le Séminaire. Je regrette donc beaucoup que ce Monsieur m'ait mis dans une aussi triste nécessité. Le Séminaire recevra sous peu une copie fidèle de la présente, ${ }^{27}$ pour qu'il puisse y répondre en s'adressant directement à Rome, car il me paraît inutile d'entrer en discussion avec $\mathrm{M}$. Baile, comme le demande Votre Excellence, parce que c'est avec le Saint-Siège que le Séminaire a toujours voulu traiter directement de l'affaire de sa cure. Et, en effet, lorsqu'en 1830, l'évêque voulut régler cette affaire sur les lieux, le Supérieur lui signifia qu'on en appelait à Rome. ${ }^{28}$ En avril dernier, je fis tout mon possible pour engager M. Granet et M. Baile à s'entendre ici amicalement avec l'évêque, pour soumettre ensuite d'un commun accord notre mutuel arrangement; et comme, pour toute

27 ACM, Lettres de Mgr Bourget, 13: 179-186. A.M. Dominique Granet, supérieur du séminaire, 30 octobre 1863 : "Je vous adresse, avec la présente, une copie de la lettre que j'ai adressée, le 29 septembre dernier à S.Em. le card. Préf. de la S. Cong. de la Propagande, comme je vous l'avais promis le 30 avril de cette année."

28 Voir plus haut, note 14 . 
réponse, M. Granet me demanda, le 28 de ce même mois d'avril, le statu quo dont il est aujourd'hui question, en ajoutant que le Séminaire prendra en sérieuse considération ce que je lui dirai, et que s'il peut y accéder il le fera de bonne volonté, je lui répondis que, dans ce cas, il valait mieux que $M$. Baile allât plaider la cause du Séminaire à Rome. Car il me paraissait impossible de céder au Séminaire un statu quo qui n'est pas défini, et il ne me paraissait pas convenable de m'exposer à des refus de la part de mes inférieurs. M. le Supérieur me répondit le 6 mai suivant qu'en effet, il serait bien malaisé d'envoyer à la Propagande des demandes communes; et en conséquence, M. Baile partit pour la Ville Eternelle, muni d'une excellente lettre de recommandation de ma part et appuyé de toute l'influence de son Supérieur-général, et sans que j'aie écrit un mot pour entraver ses négociations. Votre Eminence aura donc pour agréable que je n'intervienne plus dans cette affaire, autrement qu'en lui donnant les explications qu'Elle jugera à propos de me demander.

Il apporte ensuite aux affirmations de MM. Carrière et Baile les correctifs qu'il croit opportuns; il énumère les craintes que lui inspire l'approbation d'un statu quo mal défini; il dit la pensée qui le guide:

J'admets que le Supérieur de St-Sulpice soit curé à perpétuité de la paroisse de Montréal. Mais en même temps, j'entends qu'il soit dans l'entière dépendance de l'évếque. Cette entière dépendance de l'évêque chez le curé de Montréal, doit être celle que requièrent les saints canons pour le bon gouvernement des âmes. Ce que je désire souverainement, c'est que tout le diocèse soit régi selon les règles canoniques et que toutes les paroisses qui le composent, et surtout celle de Montréal, qui est la plus importante, et qui pour cela, doit être le modèle de toutes les autres, soient administrée si bien que l'on puisse dire que c'est le droit canon qui y fait tout mouvoir, et non l'arbitraire. ${ }^{29}$

29 ACM, Lettres de Mgr Bourget, 13: 116-171. 
Le combat est engagé. Comment se déroulera-t-il ? Et quelle en sera l'issue?

\section{III}

\section{LE VOYAGE DE MONSEIGNEUR BOURGET À ROME}

Le 18 juillet 1864, Mgr Bourget demandait au cardinal Barnabo la permission de se rendre à Rome. Entre tant de raisons qui justifiaient le voyage, le problème de la Paroisse n'était pas le moindre; et il n'y voyait de solution qu'en des conférences entre les parties intéressées et qui se tiendraient à Rome, sous les yeux de la Propagande. Car si, "après tout ce qui s'est passé, nous entrons en discussion sans aucun intermédiaire qui, à chaque question difficile qui va immanquablement surgir, puisse nous montrer les droits que la Sainte Église a assuriés à ses évêques et à ses curés, nous ne ferons qu'embrouiller les choses." 30 Seuls des intérêts d'ordre majeur pouvaient inspirer ce voyage à $\mathrm{Mgr}$ Bourget. Sujet plus que d'autres au mal de mer, il était revenu de Rome deux ans plus tôt fortement ébranlé dans sa santé. ${ }^{31}$ Cependant il s'était remis à la besogne assez tôt, et avec une ardeur que Mgr Baillargeon, archevêque de Québec, ne savait assez admirer ${ }^{32}$ Mais il avait trop présumé de ses forces. Entre 1862 et 1864, les séjours à l'Hôtel-Dieu

30 ACM, Mgr Bourget. Lettres pers. Voyage à Rome, 1864-1865. La lettre est datée de Kingston.

31 ACM, Lettres de Mgr Bourget, 12: 536. Au cardinal Barnabo, 19 août 1862 ... La traversée sur la mer a été tout à fait défavorable à ma santé. Comme je crains d'avoir contracté une maladie grave, et qu'il pourrait bien plaire à Dieu de disposer bientôt de mes jours, je crois de mon devoir de solliciter d'avance pour le Grand Vicaire que le chapitre nommera, advenant mon décès, tous les pouvoirs papaux communiqués à l'évêque, avec la faculté de déléguer ceux que l'évêque lui-même peut déléguer." - Le 22 novembre 1862, le Vicaire général, M. Truteau, prescrivait des prières pour le retour à la santé de Mgr Bourget. Mand. des évêques de Montréal, 4: 335.

$32 \mathrm{AAQ}$, Registre des Lettres, no 27: 539. Mgr Baillargeon au cardinal Barnabo, Québec, 16 octobre 1862: “... Il (Mgr Bourget) a été très malade et longtemps en danger. Il n'est pas encore parfaitement rétabli et il est probable qu'il ne se rétablira jamais bien. Cependant, tout faible qu'il est encore, à la suite de cette maladie, il a repris le cours de ses travaux et s'y livre avec son ardeur accoutumée. Je crois qu'il y a bien des saints dans le ciel que l'Eglise honore d'un culte public, qui n'en ont pas fait tant que lui..." 
avaient été fréquents et parfois prolongés. En fait, la convalescence n'était pas encore terminée quand le 14 novembre, il quittait Montréal, accompagné de M. Norbert Lavallée, curé de SaintVincent-de-Paul et de son médecin, le docteur Louis-Benjamin Durocher.

Pour accélérer la solution du problème, dans un long mémoire, adressé le 9 novembre, au cardinal Barnabo, il avait clairement établi ses positions. Le statu quo que demandait le Séminaire contenait des éléments louables et des éléments condamnables. Il approuvait les premiers, réprouvait les seconds. Il ne contestait pas le droit du Supérieur à devenir curé de VilleMarie; mais il entendait qu'il le devînt en vertu d'une lettre de l'Ordinaire: car il se considérait comme seul autorisé à déléguer à tous les prêtres du diocèse, même aux messieurs du Séminaire, les pouvoirs ad curam animarum. Il conteste cette interprétation du statu quo selon laquelle il a été impossible jusqu'ici de démembrer l'immense paroisse de Notre-Dame. Sur le grand argument du Séminaire, savoir, la lettre du cardinal Pedicini, du 19 décembre 1831, il écrit:

Il est évident à la seule inspection de cet important rescrit, 1) que les évêques qui ont accordé au Séminaire les privilèges dont il a joui paisiblement pendant 131 ans, n'ont pu ni diminuer ni lier l'autorité de leurs successeurs; 2) qu'à la vérité, cette longue possession du Séminaire avait son poids, mais ne lui donnait pas un droit strict, puisque la S. Cong. ne croit pas devoir faire autre chose que de conseiller aux deux parties d'en venir à un accord mutuel; 3) que la sanction pontificale n'a été ajoutée au décret de la S. Congr. que pour confirmer le fait des évêques de Québec, prédécesseurs de celui qui revendiquait le droit que lui donnaient les Saints canons. ${ }^{33}$

Le Mémoire est un document substantiel, solide, qui fait appel tour à tour aux canonistes de grand renom.

Le 11 novembre, il écrivait à M. Granet qu'il lui en destinait une copie. Il allait à Rome, affirmait-il, non pour accuser qui

33 ACM, Lettres de Mgr Bourget, 14: 162 et ss. 
que ce soit, mais pour défendre les droits de l'évêque. Il espère que le Séminaire sera représenté devant la Propagande par des procureurs munis de pleins pouvoirs. ${ }^{34}$ Son vœu devait être exaucé, car la Minerve du 21 novembre annonçait le départ pour Rome de M. Joseph Bayle, p.s.s.; et celui-ci aurait pour collaborateur nul autre que l'histurien du Montréal héroïque, M. Étienne-Michel Faillon, supérieur de la Procure de Saint-Sulpice, récemment établie dans la Ville-Eternelle. ${ }^{35}$

Le soir même de son arrivée à Rome, le 11 décembre, $\mathrm{Mgr}$ Bourget était reçu par le cardinal Barnabo. Celui-ci, de son propre chef, aborde tous les problèmes chers à l'évêque, et il se montre sympathique, encourageant. Il dit "que pour ce qui regarde la cure de Montréal, le Concile de Trente donne à l'évêque le pouvoir de la gouverner, de la diviser, etc. pour le bien des âmes, et qu'il avait parlé dans ce sens à $M$. Carrière: que le Supérieur du Séminaire qui, par le seul fait de sa nomination, devient curé de cette paroisse, doit se pourvoir des provisions qui lui sont nécessaires pour remplir ses fonctions curiales; que M. Faillon lui a dit que le nouveau supérieur de St-Sulpice sera plus d'accommodement que $M$. Carrière, qui n'entrait pas aisément dans les règles communes du droit"'.36 On sent à le lire qu'il est heureux, optimiste. Il ne soupçonnait pas, en ce moment, que le problème de la cure de Montréal le retiendrait en Europe pendant un an.

Ce n'est pas inutilement qu'il s'était fait accompagner de son médecin. Le 13 décembre, deux jours après son arrivée, il écrit:

Me voici avec une fièvre assez forte, accompagnée de maux de tête. Ce ne sera rien, et le $\mathrm{D}^{\mathrm{r}}$ Durocher

34 Ibid: 155-161.

35 En 1860, la Sacrée Congrégation des Evêques et Reguliers, approuvait la Compagnie des prêtres de Saint-Sulpice et demandait que, "conformément à ce que pratiquaient les diverses congrégations qui n'avaient pas leur supérieur général à Rome, la Compagnie y ait un procureur général auprès du Saint-Siège, pour y traiter les affaires qui pourraient survenir." M. Faillon fut le premier à occuper le poste et il arrivait à Rome en mai 1864. Vie de M. Faillon, prêtre de Saint-Sulpice (Paris, 1877), 280.

36 ACM, Mgr Bourget, Lettres pers. Voyage à Rome, 1864-1865. A M. Truteau, "adm. 
est à l'œuvre pour me soigner. Cela ne vaut pas la peine d'être mentionné; mais vous comprendrez par là que quand je dis que je suis bien, il faut me donner toute confiance. ${ }^{37}$

Dans son Journal de voyage, ${ }^{38}$ où il note, en style télégraphique, ses occupations de chaque jour, on voit qu'il n'oublie pas longtemps la cure de Montréal. On ne risque pas de se tromper en affirmant que ses visites au célèbre $P$. Perrone, s.j. ne sont pas de simples visites de politesse, qu'il y est question de théologie et de droit canon. Le 14 janvier 1865, il écrit à M. J.-O. Paré:

J'ai vu plusieurs fois M. Baile depuis son arrivée à Rome; et il m'a dit qu'il attendait de Montréal des renseignements pour procéder dans l'affaire qu'il est chargé de traiter au nom de sa maison. Je suis bien décidé à avoir avec lui et avec ses confrères les meilleurs rapports: car je voudrais que la paix et la confiance puissent régner, après que ce différend sera terminé. ${ }^{39}$

Le 15 février, il reçoit copie du mémoire présenté à la Propagande par MM. Faillon et Baile. Il l'apprécie brièvement ainsi :

Le mémoire est écrit en latin et est très long ( 85 pages). Il consiste en un monitum, dans cinq questions et dans une ou plusieurs conclusions. Il est facile de s'apercevoir qu'il est l'ouvrage de théologiens accoutumés à la forme scolastique. Il s'y trouve aussi une bonne tirade sur l'histoire de l'Eglise du Canada, dont M. Faillon, signataire de ce mémoire ainsi que M. Baile, s'occupe aussi activement que le lui permettent ses infirmités. ${ }^{40}$

Homme d'Église, Mgr Bourget parlait, écrivait et lisait le latin avec facilité. Si donc la lecture du document provoque en

37 Ibid., à M. J.-O. Paré, secrétaire.

38 Ibid., Lettres pers. 1860-1862. Ce journal écrit de la main de Mgr Bourget fait suite, dans un petit cahier non cousu, aux buts du voyage de 1862.

39 ACM, Mgr Bourget, Lettres pers. Voyage à Rome 1864-1865. A M. J.-O. Paré.

40 Ibid., A M. Truteau, adm. 21 février 1865. - Copie du Mémoire dans $A C M$. Paroisse de Notre-Dame, 1678-1865, no 8. 
lui un malaise et une impatience qui montent parfois jusqu'à l'indignation, il ne faut pas s'en prendre à la langue dans laquelle il est écrit, mais à son contenu, à l'esprit qui l'anime, à la demande qu'il réitère: confirmation du statu quo qui le soustrait à l'autorité de l'Ordinaire. Il y relève de plus des entorses à l'histoire, à cette histoire pénible qu'il a lui-même vécue depuis plus de 40 ans à Montréal. L'antagonisme qu'il voit partout entre le Séminaire et l'évêché, les Messieurs ne le voient nulle part, ou du moins ils en rejettent toute la responsabilité sur l'évêque. Le monitum qui sert d'introduction est, selon le sens du mot, un avertissement, une mise en garde à l'adresse des cardinaux qui auront à trancher le débat. Les prêtres du séminaire, y est-il dit, ne sont pas comme les religieux liés par un vœu d'obéissance. Ce qui les retient à Montréal, c'est la certitude qu'ils font œuvre utile dans l'esprit et selon les règles de leur vénéré fondateur, M. Olier. Que l'un ou l'autre décide d'aller se dévouer ailleurs, il n'est rien, il n'est personne qui puisse le retenir. Sa liberté est entière. Or, s'il était permis à l'évêque d'intervenir dans l'administration de la Paroisse comme il l'entend, il serait à craindre que le découragement s'empare des Messieurs et que l'un après l'autre, ils rentrent en France. Et qu'arriverait-il alors ? Montréal serait privé du ministère de 57 prêtres; de plus, par suite de la dissolution du Séminaire, ses biens, soutien de tant de bonnes œuvres, deviendraient la propriété de l'État: ainsi le veut la loi civile de 1840 incorporant la communauté. ${ }^{41}$ Pour le reste, le document

${ }^{41}$ Le monitum se terminait par une protestation d'obéissance humble et soumise au cas où la Propagande jugerait nécessaire de retirer les Sulpiciens de Montréal. Mais avant d'en venir là, les cardinaux étaient priés de lire la 2e partie de l'Histoire de la Colonie française en Canada, dont M. Faillon corrigeait alors les épreuves: "Ut autem ipsa (Congrãtio) judicare possit utrum vere Deus nos ad ministerium Marianapoli exercendum vocaverit, et an nobis hucusque justa fuerit ita sentiendi causa; obsecramus S. Cong. ut antequam decernat, ea comperta sibi faciat quod quæ de fundatione Marianapolis in II art. Part. hist. Coloniæ gallicæ in Can. (quod nunc et quidem opportunissime typis mandatur). Adamussim relata sunt et ut ista habeantur quasi explicationes et appendices hujusce documenti, quod ipsius oculis subjicere præsumimus." - N'exagérons pas: l'Histoire de la Colonie française n'a pas été rédigée pour soutenir les prétentions du séminaire contre l'évêque. Dès 1850 , M. Faillon rêvait de raconter nos origines religieuses si belles, mais si peu connues alors (RHAF, (1957) : 107-110). Pendant 15 ans il a dépouillé inlassablement les dépôts d'archives de la France et du Canada; il a publié les biographies des grandes figures religieuses du Montréal mystique, qu'il connaissait 
n'apportait rien de véritablement neuf : il réaffirmait et développait plus longuement le mémoire présenté en 1863 par MM. Carrière et Baile, savoir: 1) la division de la paroisse ne paraît pas justifiée, parce que l'organisation du ministère pastoral répond aux besoins des fidèles; 2) le démembrement entraînerait des inconvénients si nombreux et si graves qu'il faut, à tout prix, l'éviter;3) Le moyen d'apporter remède aux maux dont se plaint l'évêque ? Le maintien du statu quo, c'est-à-dire de l'ordre de choses deux fois séculaire et consacré par la lettre du cardinal Pedicini (1831). Mgr Bourget affirmait que l'expression statu $q u o$ était vague, mal définie. MM. Faillon et Baile en donnent ici le contenu: 8 points précis, qui ne laissent place à aucune échappatoire et qui sont autant de restrictions aux prétentions de l'évêque. A celui-ci, pour ne citer qu'un exemple, il serait interdit d'introduire une communauté religieuse dans les limites de la Paroisse sans avoir obtenu d'abord le consentement du curé!

Une réponse s'imposait, et Mgr Bourget n'y manque pas. "Il faut être à l'œuvre de bon matin, écrit-il: car pour le soir je suis si fatigué, que je ne puis faire autre chose que quelques petits bouts de prière, au milieu de beaucoup de divagations d'esprit occasionnées par de continuels retours de l'âme sur ces affaires distrayantes." 42

La Réplique de Mgr Bourget est du 18 février; elle commence par des considérations sur le monitum. Pour réduire celui-ci

plus que tout autre. L'Histoire apparaît comme le couronnement de ses travaux et recherches sur le Canada. Procureur de sa communauté à Rome, il avait pour tâche de défendre celle-ci auprès du Saint-Siège. Il a pu s'exagérer la valeur probante de son Histoire: il y voyait un argument en faveur du Séminaire, et il le présente comme tel. Mais son intention était bonne. - Quant aux dangers de voir le séminaire disparaître, ses sujets rentrer en France et ses biens faire retour à la Couronne, Mgr Bourget ne les a jamais considérés comme sérieux. A ses yeux, c'étaient exactement ce que les philosophes appellent des futuribles c.e. des choses qui arriveraient, si telles conditions étaient posées, mais qui n'arriveront pas, parce que les conditions ne seront pas posées. Les textes que nous citons dans cet article prouvent à l'évidence qu'il voulait garder les Messieurs, qu'il tenait à les garder à Montréal. "Ce sont de bons prêtres: que fèrions-nous, si nous ne les avions pas ?" écrivait-il, un jour, à un détracteur du Séminaire.

42 ACM, Mgr Bourget, Lettres pers. Voyage à Rome, 1864-1865. A M. Truteau, adm. 15 février 1865. 
à ses justes proportions, écrit-il, il suffit de se rappeler ce que demande l'évêque de Montréal. Que veut-il donc ? L'exercice de ses droits, lequel est parfaitement compatible avec les légitimes privilèges du séminaire qui ne sont d'ailleurs pas contestés.

Il répond ensuite aux reproches qu'on lui adresse. Ce qu'on appelle abus de pouvoir, violations des intangibles droits du Séminaire, lui a été dicté par sa conscience: car, Ordinaire du lieu, il a charge d'âmes, à Montréal comme ailleurs. Ses initiatives avaient pour but, non de jeter le blâme et le discrédit sur le Séminaire, mais de suppléer à son insuffisance dans une ville qui croît rapidement, et qui est, de plus, le centre et l'âme du diocèse.

Les choses auraient pu en rester là. Mais, pleins de leur sujet, MM. Faillon et Baile adressent aux cardinaux un autre long mémoire latin, le 22 avril, 43 auquel l'évêque répond par des observations le 29.41 Tout cela se fait dans le temps. Áu début de mai, il reste encore une pièce à venir, et non la moindre: l'avis du Consulteur de la Propagande, c'est-à-dire l'analyse du dossier et son jugement sur l'affaire. Il faut ensuite imprimer les documents, les réunir en fascicules, les distribuer aux cardinaux, accorder à ceux-ci le temps requis pour les étudier avant de former leur jugement. A la mi-juin, on prévoit que le problème sera soumis à l'assemblée plénière de septembre, avant les vacances d'automne.

\section{IV}

\section{LE SEJOUR DE MONSEIGNEUR BOURGET EN FRANCE}

Mais il fait chaud et humide à Rome. Depuis le 11 décembre 1864, date de son arrivée dans la Ville-Eternelle, Mgr Bourget a travaillé d'arrache-pied, malgré la fièvre et les maux de tête qui l'ont plus d'une fois abattu: car la cure de Montréal n'est par son unique problème. Il a hâte d'en finir: il éprouve le besoin de partir, d'aller respirer sous d'autres cieux. Pour surveiller ses intérêts à Rome, il laisse M. Lavallée, et il se rend en France.

43 ACM, Paroisse de Notre-Dame, 1678-1865, no 14.

44 Ibid., no 15. 
Il choisit, ou plus exactement, on lui assigne comme lieu de repos l'Hôtel-Dieu de La Flèche. Il y arrive plus mort que vif, le 7 juillet. La supérieure, Mère Gaudin, a tôt fait de constater que l'évêque est malade, très malade: fièvre prononcée qui revient immanquablement tous les soirs; voix couverte, qui a peine à se faire entendre, maux de tête, faiblesse générale. Il aurait besoin d'un grand repos, ce repos absolu si cher à la Faculté.

Mais l'expérience a appris à la Mère Gaudin qu'aux tempéraments dynamiques, le repos absolu n'est pas le remède le plus approprié. Ce serait l'achever, écrit-elle, que de le condamner à l'inaction. Et puis, confiance: "Dieu le conservera, je l'espère, lui seul le fait depuis des années, il le fera bien encore."45 Avec la permission de Mgr Fillion, évêque de Mans, il remplace l'aumônier en congé de vacances; il célèbre la messe de communauté; entend les confessions des religieuses, mais "promptement, c'est ainsi convenu avec mes Filles", leur fait de courtes instructions, reçoit quelques visiteurs, vaque à sa correspondance qui n'est pas considérable, car seuls M. Lavallée et le Dr Durocher savent où il est. ${ }^{46}$

Après un mois de ce régime, il se rend en Belgique, où l'appellent les intérêts du diocèse; et de là il reviendra à Paris. Mais pourquoi Paris ? Si malade qu'il fut à son départ de Rome, il a tout prévu. En son absence, la Propagande apportera une solution au problème de Montréal; et comme d'avance il l'accepte, dès qu'elle sera de droit public, il reviendra dare-dare au pays. Car il a hâte de revoir ses enfants. ${ }^{47}$ Mais il se peut aussi que la Propagande ne tranche pas si vite le débat et que sa présence soit encore utile à Rome. Dans ce cas. il y retournera: et c'est à

45 Arch. de l'Hôtel-Dieu de Montréal. Lettre de la Mère Gaudin, La Flèche, 20 juillet 1865.

46 Ibid.

47 Dans la seconde Pastorale sur le démembrement de la Paroisse, 23 mai 1866, il parle ainsi de sa longue absence du diocèse: "C'est également pour l'amour que Nous vous portons à tous, que Nous nous sommes privé pendant plus de treize mois, des douceurs de la patrie et du bonheur qu'il y a pour tout pasteur de se trouver au milieu de son troupeau. Or, ces longues et fréquentes absences qu'il Nous a fallu faire pour le bien du diocèse en général et le vôtre en particulier, Nous ont été plus pénibles que vous ne sauriez le croire." Mand. des év. de Montréal, 5: 154. 
Paris, Hôtel du Bon La Fontaine, rue de Grenelle, qu'on pourra le rejoindre..$^{48}$ Le 19 septembre, il recevait de M. Lavallée une dépêche télégraphique ainsi conçue: "Le cardinal vous demande absolument à Rome." ${ }^{49}$ Que s'était-il donc passé ? A sa réunion plénière du 18 septembre, la Propagande avait cru que l'heure n'était pas encore venue de régler le problème par voie d'autorité. Elle souhaitait une solution pacifique, agréée par le Séminaire et par l'évêque avant d'être confirmée par le Pape. En conséquence, l'évêque de Montréal et le supérieur général de Saint-Sulpice, M. Caval, sont convoqués à Rome où en présence de trois cardinaux délégués à cette fin, ils tenteront d'ajuster leurs différends.

\section{V}

\section{DE RETOUR À ROME POUR LE DERNIER COMBAT}

Mgrr Boürget écrivait à M. J.-O. Paré, le 20 septembre: "Je reprends aujourd'hui avec $M$. Huberdault la route de la VilleÉternelle, où je ne serai tout juste que le temps nécessaire pour terminer les affaires pour lesquelles on nous demande. Je suis en bonne santé ainsi que mon compagnon de voyage, et nous sommes l'un et l'autre contents et résignés à la sainte volonté de Dieu." 50

Malade ou bien portant, l'évêque de Montréal reste soumis aux lois des pays qu'il traverse. Or tous les voyageurs qui se dirigent vers Rome sont immobilisés à Livourne par la quarantaine. L'évêque en profite pour rédiger un document sur la cure de Montréal, dont la substance se trouve également dans une lettre au cardinal Barnabo, qui porte la date du 25 septembre 1865 :

Ce que demande l'évêque dans cette affaire est de pouvoir gouverner la cure de Montréal, comme tou-

48 ACM, Mgr Bourget, Lettres pers. Voyage à Rome, 1864-1865. Au cardinal Barnabo, Paris, 9 septembre 1865. Il compte partir sans retard pour le Canada. Il ajoute en P.S.: "Si V. Em. juge à propos de m'écrire avant quinze jours, que je me propose de quitter la France, mon adresse est: Paris, Hôtel du Bon La Fontaine, Rue Grenelle, St-Germain, 16."

49 Ibid., A M. Paré, Paris, 20 septembre 1865.

50 Ibid. 
tes les autres de son diocèse, selon les règles canoniques.

Comme cette demande est juste et nécessaire, il se croit obligé de faire instance auprès du St-Siège jusqu'à ce qu'il l'ait obtenue, car sans cela il n'y aura jamais dans le diocèse que trouble et division: tandis que, l'on ne saurait en douter, la paix y serait bientôt rétablie et s'y maintiendrait avec bonheur, si chacun, usant des droits que lui accorde la Sainte Eglise, se tenait à sa place et y remplissait fidèlement ses devoirs.

Après un rappel historique du problème, il conclut:

En divisant maintenant la paroisse de Montréal, on multiplie la sollicitude pastorale, on régularise le service divin, on établit une noble émulation entre toutes les cures dans le travail à faire pour y combattre les mauvais principes du temps et y affermir le règne des vertus: et on prévient les dangers du schisme... Quand même les privilèges de St-Sulpice seraient apostoliques, il serait temps de les révoquer, parce qu'ils peuvent que nuire au bien des âmes. ${ }^{51}$

Le 3 octobre, il écrivait à M. Lavallée: "Je vis hier le cardinal Barnabo qui me dit que la $\mathrm{S}$. Congrégation avait admis en principe que tout ce que je demandais était conforme aux saints canons; mais que, pour le bien de la paix, il fallait faire quelques concessions." ${ }_{22}$ Pie IX lui avait tenu le même langage: "Le droit est pour vous, mais l'usage est pour le Séminaire; mais tout va s'arranger pour le mieux." 53

L'évêque se demande ce qu'il pourrait concéder au Séminaire, sans déroger aux lois de l'Église: il prie, il réfléchit, il consulte les théologiens. Quand la lumière s'est faite dans son esprit, il signifie à M. Caval son désir d'entrer en pourparlers. ${ }^{54}$ Celui-ci lui fait tenir aussitôt des observations, qui paraissent fort encou-

51 Ibid., Au cardinal Barnabo, Livourne, 25 septembre 1865.

52 Ibid.

53 Ibid., A M. Truteau, Rome, 4 octobre 1865.

54 ACM, Paroisse de Notre-Dame, 1678-1865, no 16. Minute d'une lettre de l'Evêque de Montréal à M. Caval, 7 octobre 1865. 
rageantes, le Séminaire renonçant spontanément à plusieurs des points en litige: approbation par l'évêque de tous les Messieurs qui viendront au Séminaire; la Paroisse sera désormais dévolue non au supérieur, mais à la communauté et l'évêque donnera l'investiture canonique au sujet que le supérieur présentera comme curé, etc. ${ }^{55}$

Une seule difficulté n'était pas aplanie mais elle était de taille: le mode de révocation du curé. Le problème n'était pas nouveau, et il existait des principes de solution. Dans la bulle Firmandis, Benoit XIV avait établi le droit relatif aux paroisses, dont le curé appartenait à un institut religieux: et il avait statué que le supérieur religieux et l'Ordinaire du lieu avaient le même droit de révoquer un tel curé, sans que l'un soit tenu de justifier son geste devant l'autre. Tel était le droit général. Mais il y avait à cela une exception. S'agissait-il de la révocation d'un curé vivant habitueliement sous le même toit que son supérieur général, le droit de l'évêque était limité; il devait, dans ce cas, obtenir l'assentiment du supérieur général, et à son défaut, recourir au Saint-Siège. C'était cette exception que réclamait M. Caval en faveur du curé de la Paroisse. Il la croyait nécessaire à la vie et à la survie du séminaire de Montréal, nécessaire - le mot est écrit en toutes lettres nécessaire pour soustraire celui-ci à l'arbitraire. ${ }^{56}$

Mais à quoi pensait donc M. Caval ? Sur quoi se basaient ses craintes ? Pour le bien comprendre, un rappel historique nous paraît ici nécessaire. En 1846, Mgr Bourget avait exercé une véritable pression morale pour amener $M$. Joseph-Vincent Quiblier à ne pas se laisser réélire au poste de supérieur qu'il occupait depuis quinze ans. Il l'exhortait, en même temps, à rentrer en France. M. Quiblier restant sourd à ce dernier

55 Ibid., M. Caval à Mgr Bourget, 7 oct. 1865.

56 ACM, Mgr Bourget, Lettres pers. Voyage à Rome, 1864-1865. A M. Paré, Rome 17 octobre 1865. Mgr Bourget transmet à son correspondant une copie de la lettre de $M$. Caval. Voici le passage qui nous intéresse en ce moment: "Le supérieur de St-Sulpice pense que sa communauté de Montréal ne pourrait pas subsister longtemps, et que d'ailleurs il ne trouverait pas de sujets qui voulussent s'expatrier pour se consacrer à cette mission, s'ils n'avaient quelque garantie qui les mît à l'abri des actes arbitraires au sujet des révocations." 
conseil, l'évêque lui avait enlevé ses lettres de grand-vicaire ainsi que la direction des religieuses de la congrégation et lui avait enfin signifié qu'il n'avait plus besoin de ses services dans le diocèse. ${ }^{57}$

L'affaire avait fait du bruit et semé la division au Séminaire, dans le clergé, à la ville: car le prestige de M. Quiblier était immense, et rien ne paraissait justifier les rigueurs de l'évêque à son endroit. Plus encore: pour mettre fin au scan. dale, Mgr Bourget s'était cru obligé en conscience d'interdire aux Messieurs, et cela sous peine de suspense, tout geste ou toute parole qui pût fomenter la division. ${ }^{58}$ Le Supérieur général de Paris, M. de Courson, avait vu dans l'intervention de Mgr Bourget une atteinte à la liberté des électeurs du Séminaire.

Il n'est donc pas étonnant que le mémoire présenté à la Propagande par MM. Faillon et Baile, le 15 février 1865, ait fait état du cas de M. Quiblier; c'était la preuve des abus d'autorité dont Mgr Bourget était capable, et une raison pour le Séminaire de vouloir se soustraire à sa juridiction.

Mis en cause, l'évêque se contenta d'exposer les faits dans sa Réplique, qui fut communiquée, il va sans dire, aux procureurs du Séminaire. Il dit la raison de son intervention, les circonstances qui l'ont accompagnée, la prudence qu'il a déployée pour éviter tout éclat, cherchant conseil dans cette affaire délicate, non auprès de ses chanoines, mais auprès de quatre membres du Séminaire parmi les plus remarquables; 59 il note qu'après le départ de M. Quiblier pour la France, tout rentra dans l'ordre et il conclut: "En procédant de la sorte l'évêque de Montréal crut alors et croit encore aujourd'hui avoir rendu un vrai service à St-Sulpice: et voilà qu'aujourd'hui, on lui en fait un crime à Rome." Simple façon de dire que sur un fait

${ }^{57} \mathrm{ACM}, 2 e$ voyage de l'Evêque en Europe, 1846-1847. Mgr Bourget à M. de Courson, supérieur-général, Paris, 21 avril 1847.

58 Archives de Saint-Sulpice, Séminaire et évêché. Dossier QuiblierBourget. Le document est du 11 juin 1846.

$59 \mathrm{M}$. Pierre-Louis Billaudèle, qui succéda à M. Quiblier comme supérieur, M. Armand de Charbonnel, futur évêque de Toronto, M. JosephAlexandre Baile, signataire des mémoires présentés par le Séminaire à la Propagande en 1863 et en 1865 , et $M$. Léonard-Vincent-Léon Villeneuve. 
vieux de dix-neuf ans, Mgr Bourget a la conscience en paix. Or, c'est cela précisément qui paraissait au Séminaire une menace pour l'avenir. S'il ne regrette pas, il pourrait donc recommencer ! Et s'il lui prenait fantaisie de le faire ! Le cas de M. Quiblier, épée de Damoclès suspendue sur la tête des futurs curés de la Paroisse ! Voilà ce qu'il est bon de savoir pour mieux comprendre la suite des événements.

Car Mgr Bourget pensait qu'il ne pouvait accéder à la demande de M. Caval. Et ce refus allait tout remettre en cause. A partir de ce jour, en effet, M. Caval cesse de correspondre avec lui et traite directement avec les cardinaux conciliateurs, avec le cardinal Barnabo surtout.60 Par celui-ci, Mgr Bourget sait où en sont les choses à la date du 17 octobre: il énumère 27 exigences du Séminaire, et menace de quitter Montréal, si on ne lui accorde pas la liberté qu'il réclame. ${ }^{61}$

Une semaine plus tard, la situation est plus grave encore. Il écrit:

Depuis ma dernière (...) l'affaire de la cure de Montréal a fait un pas, en ce sens que M. le Supérieur Général de St-Sulpice, ayant signifié au card. préfet qu'il ne pouvait accepter toutes mes propositions de conciliation (...), la procédure se trouve forclose. Mais les cardinaux, chargés d'entendre les parties contendantes, sont maintenant dans l'obligation, vu qu'elles n'ont pu s'entendre sur plusieurs points, de référer toute cette affaire à la première Congrégation qui se tiendra là-dessus.

Et il se pourrait, ajoute-t-il, que le mois de février me trouve encore à Rome. ${ }^{62}$ Le 30 octobre il a du neuf. La fameuse lettre du cardinal Pedicini disparaissait du dossier: il n'en serait plus question. Le cardinal Barnabo en avait donné, et aux procureurs du séminaire, s'il vous plaît, une interprétation authentique et finale. Tout le sens de ce document, avait-il

$60 \mathrm{ACM}, \mathrm{Mgr}$ Bourget, Lettres pers. Voyage à Rome, 1864-1865. Au doyen du Chapitre, Rome, 17 octobre 1865.

61 Ibid.

62 Ibid., A M. Truteau, Rome, 24 octobre 1865. 
dit en substance, est contenu en ces mots: Placeat de concordia, c'est-à-dire entente à intervenir entre les parties intéressées: c'est cela également, et rien autre chose, que Grégoire XVI avait approuvé. Or, l'entente désirée, demandée, n'a jamais existé : donc ... ${ }^{63}$ Donc, tout est à reprendre, comme si la lettre du 19 décembre 1831 n'avait jamais existé.

Et maintenant les événements vont se précipiter. Jusqu'ici les cardinaux de la conciliation se sont contentés de lire les textes écrits qui leur étaient soumis de part et d'autre. Ils décident qu'il y aura, en leur présence, affrontement des parties. $\mathrm{Mgr}$ Bourget en est averti le 2 novembre, et il se prépare au grand événement:

Je commençai par la prière et la lecture d'un chapitre de l'Imitation (...) Je tombai heureusement sur le chapitre II du livre II: de humili submissione: et je fus divinement fortifié de ces paroles: non magni pendas quis pro te contra te sit: sed hoc age UT Deus tecum sit in omni re quam facis." ${ }_{64}$

Pour être sûr de ne rien oublier dans la chaleur de la discussion, il inscrit sur une feuille, concedenda Seminario, les concessions qu'il peut faire, et sur une autre feuille, neganda Seminario, les articles qui lui paraissent inadmissibles. ${ }^{65}$

L'affrontement avait été fixé au 3 novembre, à 5 h. 30 de relevée. Sont là: les trois cardinaux, Barnabo, Caterini, Reisach, ainsi que le secrétaire de la Propagande, Mgr Capalti; MM. Caval, Faillon et Baile; Mgr Bourget. Dans ses lettres du 7 et du 14 novembre, celui-ci nous a laissé un récit détaillé de l'événement. ${ }^{66}$ Contentons-nous d'en retenir l'essentiel. M. Caval resta sur ses positions: si on ne lui accorde ce qu'il demande relativement à la révocation du curé de la Paroisse, il

63 Ibid., A M. Paré, Rome, 30 octobre 1865.

64 Traduction rythmique de l'abbé de Cigala: "Ne vous souciez pas qu'on soit pour ou contre; mais agissez toujours, de façon telle, en tout, que Dieu soit avec vous en tout ce que vous ferez."

65 ACM, Mgr Bourget, Lettres pers. Voyage à Rome, 1864-1865. A M. Paré, 7 novembre 1865.

$66 \mathrm{ACM}, \mathrm{Mgr}$ Bourget, Lettres pers. Voyage à Rome, 1864-1865. A M. Truteau. 
est bien décidé à retirer tous ses sujets de Montréal. Le cardinal Barnabo lui rappelle que le Saint-Père verrait la chose d'un fort mauvais œil : car il veut que les Sulpiciens restent à Montréal, où ils font beaucoup de bien. Mais il a dit: Placeat de concordia. Et M. Caval est prié de penser sérieusement aux conséquences du geste qu'il s'apprête à poser. Le supérieur général répond qu'il serait désavoué par ses conseillers, s'il cédait. Il demande la permission de les consulter. "Faites, lui dit le cardinal Barnabo; allez à Paris, ou écrivez à vos conseillers." Et l'assemblée est levée dans une lourde atmosphère de déception: car personne n'y trouve son compte, ni les cardinaux, dont la mission aboutit à un échec, ni les procureurs du Séminaire dont les vœux ne sont pas exaucés, ni Mgr Bourget qui se demande quand et comment tout cela finira.

La nuit est sage conseillère. Et le lendemain, 4 novembre, M. Caval faisait tenir au cardinal Barnabo la lettre suivante:

Eminence,

Je n'avais pas compris jusqu'à hier soir le véritable sens de la question sur nos affaires. Je ne pensais pas que le St-Père voulait nous obliger à rester à Montréal. Surtout, il n'avait pas pu me venir à l'esprit que nous étions placés comme devant une épreuve pour l'obéissance de St-Sulpice envers le Saint-Siège. Maintenant, après avoir entendu vos paroles si formelles, il ne peut rester aucun doute; et cette question, au point de vue du respect et de la soumission due au St-Père n'est pas susceptible de deux solutions. Pour trouver celle qui convient, je n'ai besoin que de rappeler à mon esprit les principes dont j'ai toujours fait profession et de regarder tout le passé de ma vie. Je suis certain d'ailleurs que la Congrégation tout entière souscrira sans balancer cet acte de ma sincère soumission. Veuillez, Eminence, me permettre de la faire parvenir à Sa Sainteté par Votre Eminence,

Caval.

La lettre au Pape était ainsi conçue: 


\section{Très Saint Père,}

Permettez-moi de déposer à vos pieds l'expression de nos vrais sentiments. Comme les plus dévoués de vos fils, nous serons toujours disposés à dire: Roma locuta est, causa finita est. Telle est notre doctrine, et telle sera notre conduite jusqu'à la fin. Nous nous soumettons purement et simplement aux dispositions de la bulle Firmandis touchant les révocations et tout ce qui sera selon le bon plaisir de Votre Sainteté. ${ }^{67}$

De son côté, Mgr Bourget adhérait pleinement aux décisions que prendrait la Propagande. ${ }^{68}$ L'orage était passé. Quel soulagement pour tous les intéressés !

Le 7 novembre, Mgr Bourget et $M$. Caval étaient admis ensemble auprès du Saint-Père. Ils protestèrent l'un et l'autre de leur sincère soumission et attachement au Saint-Siège. "Je dis, ajoute Mgr Bourget,

que j'étais bien éloigné de croire que, pendant mes 25 années d'administration, je n'avais jamais commis aucune faute: qu'il se rappelait sans doute que je lui en avais demandé pardon, chaque fois que j'avais eu le bonheur d'être à ses pieds, comme je le faisais encore aujourd'hui, en lui demandant pardon de toutes censures encourues et la pénitence requise pour de semblables fautes. Le Saint-Père eut alors la bonté de me dire que j'étais un bon évêque et que ma pénitence serait de vivre en bon accord avec le Séminaire. Et tout de suite, pour lui prouver ma bonne volonté, je lui demandai la permission de donner, en sa présence, le baiser de paix à M. Caval et, en sa personne, à tous les membres de sa Compagnie: ce qu'il m'accorda de bonne grâce. Je m'empressai donc d'embrasser ce Monsieur, sous les yeux du Vicaire du Christ, en preuve de l'affection sincère que je conserve pour tous ceux qui composent sa Compagnie.

Ainsi se termina cette entrevue solennelle, après laquelle M. Huberdault ayant été admis, le Saint-

${ }^{67}$ ACM, Paroisse de Notre-Dame, 1678-1865, no 32.

68 Ibid., no 33. 
Père, au lieu de sa signature qu'il lui avait demandée avec sa bénédiction, écrivit, au bas d'un magnifique portrait de Pie IX qu'il lui présentait, cette touchante sentence de l'Ecriture, qui semblait résumer tout ce qui venait de se passer sous ses yeux: Ecce quam bonum et jucumdum habitare fratres in unum ! Elle me rappellera, cette significative sentence, toutes les impressions de cette audience. ${ }^{69}$

VI

\section{LA SOLUTION}

Mgr Bourget et M. Caval pouvaient quitter Rome, et ils ne tardèrent pas à le faire. Il restait à formuler le décret, et cette tâche était dévolue à Mgr Capalti, secrétaire de la Propagande. Le texte en était approuvé par l'assemblée plénière du 2 décembre, confirmé par Pie IX huit jours plus tard et rendu püblic le 22.

Il reconnaissait à l'évêque le droit d'ériger dans les limites de la Paroisse civile de Montréal autant de paroisses canoniques qu'il jugerait bon; la paroisse Notre-Dame se voyait conférer une primauté d'honneur: toutes les paroisses à ériger devaient d'abord être offertes au Séminaire: si celui-ci les refusait, il appartiendrait à l'évêque de les pourvoir d'un clergé résident. Quant à la nomination et à la révocation du curé de Notre-Dame, on suivrait les prescriptions de la bulle Firmandis: le supérieur avait le droit de le présenter, mais il recevait son investiture de l'évêque: il pouvait être révoqué, soit par le supérieur, soit par l'évêque, sans que l'un soit tenu de s'entendre au préalable avec l'autre. Tels sont les points essentiels du décret.

Dans la pensée de la Propagande, de Mgr Bourget et de M. Caval, il devait mettre fin aux débats et ramener la paix. C'était trop espérer d'un seul coup. Son exécution souleva une autre tempête, qui devait durer près de dix ans. La pastorale de Mgr Bourget qui le promulgue est du 23 avril 1866 : et quand celui-ci donne sa démission, le 11 mai 1876 , le calme venait à peine de se rétablir.

${ }^{69}$ ACM, Mgr Bourget. Voyage à Rome, 1864-1865. A M. Truteau. 
Dans l'intervalle, l'évêque de Montréal avait, plus d'une fois, demandé d'être libéré de sa charge, pour le bien de la paix dans la province; et afin aussi de se livrer à la méditation des années éternelles avant de paraître devant le Souverain Juge. Il lui semblait qu'un évêque de Montréal plus jeune, moins engagé que lui dans les luttes des 25 dernières années, obtiendrait en moins de temps un meilleur résultat. Rome pensait, au contraire, qu'il pouvait mieux que tout autre, assurer la mise en vigueur du décret de 1865, en ce qu'il avait d'essentiel et d'urgent. Il se soumit.

\section{CONCLUSION}

L'administrateur du diocèse, M. Alexis-Frédéric Truteau, écrivait à Mgr Bourget, le 17 novembre: "Mgr Larocque, à qui nous avons communiqué votre lettre sur les prétentions des Sulpiciens, a répondu: "on voit bien que le ciel a destiné Mgr de Montréal à des combats de géant." 7o

Combat de géant, en effet, que celui entrepris par $\mathrm{Mgr}$ Bourget contre un usage approuvé tacitement par ses prédécesseurs pendant plus d'un siècle: combat qui le mettait aux prises avec une famille sacerdotale identifiée à Montréal depuis deux siècles, dont les membres, d'une pureté de vie exemplaire, s'acquittaient de leurs tâches avec dévouement, zèle, charité; qui était représentée à Rome par un homme universellement estimé, M. Faillon, et qui, en surplus, comptait d'ardents défenseurs jusque dans le monde des cardinaux..$^{71}$

Combat de géant aussi, qui avait pour but de permettre à l'Église de se développer au même rythme que la cité, en mettant en place une organisation pastorale, capable de répondre aux besoins présents et futurs.

Depuis 20 ans, le Séminaire faisait de très louables efforts pour faciliter aux fidèles de l'immense paroisse l'accomplisse-

${ }^{70}$ ACM, Lettres de Mgr Bourget, 14: 421.

71 Dans sa lettre du 14 novembre à $\mathrm{M}$. Truteau, Mgr Bourget signale que le cardinal de Villecourt avait protesté par écrit auprès du Pape contre la façon dont le cardinal Barnabo avait traité les procureurs du Séminaire, à la réunion du 3 novembre. 
ment de leurs devoirs religieux. Mais au dire de Mgr Bourget, le service paroissial restait encore inadéquat. Le rapide accroissement de la population ne pouvait que rendre la situation plus grave encore: car le Séminaire n'avait ni les ressources financières, ni le personnel requis pour construire les églises et diriger les multiples paroisses que le bien des âmes demandait à Montréal. Il était temps, grand temps que l'Ordinaire exerçât les droits que lui conférait sa charge sur la ville.

Si l'on tient compte de l'importance des intérêts en jeu, de la profonde conviction que chaque partie avait d'être dans son droit, et des fortes personnalités qui s'affrontent, il n'est pas étonnant que la dernière phase de la lutte ait été violente et que les contendants en soient venus aux insinuations de mauvaise foi.

La sagesse de la décision finale en est d'autant pius manifeste. S'élevant au-dessus de tout ce que l' "hommerie" avait jeté de passionné et d'irritant dans le débat, la Propagande retient ce qu'il y avait de bon dans les arguments des deux parties en présence. Elle affirme le droit qu'a l'évêque de diviser la paroisse et ouvre par là la voie à une sollicitude pastorale mieux adaptée aux circonstances nouvelles. Mais, en même temps, elle reconnaît, elle consacre, en quelque sorte, les immenses services que les Messieurs ont rendus à l'Église de Montréal. Et cela, de deux façons: primauté d'honneur conférée à la paroisse Notre-Dame, et qui durera jusqu'à la fin des temps: plus encore, et c'est l'obligation faite à l'évêque d'offrir d'abord au Séminaire toutes les paroisses qui surgiront dans les limites de la paroissemère; obligation qui n'est pas, elle non plus, limitée dans le temps.

Cette solution qui maintient les imprescriptibles droits de l'évêque, qui reconnaît un titre moral à des usages longtemps possédés et exercés de bonne foi par un institut de prêtres et qui contient un témoignage d'estime à l'adresse du Séminaire de Montréal est d'abord l'œuvre des cardinaux de la Propagande. Ils ont pensé au bien des âmes, sans vouloir condamner personne. 
Mgr Bourget avait pour lui le droit. Il le disait et le répétait sur tous les tons: son mérite est immense. Il n'aurait rien perdu à y mettre plus de modération: car sa cause était bonne. Il ne faudrait pas minimiser la part qui revient aux Procureurs du Séminaire. L'acte d'adhésion, écrit par M. Caval, le 4 novembre 1865, manifestait un attachement au Saint-Siège digne d'un successeur de M. Olier. Guidé par la foi, la foi nue, il acceptait ce qui, hier encore, apparaissait à sa raison comme la ruine du Séminaire de Montréal, si cher à la Compagnie de Saint-Sulpice. Au moment où il écrivait, il ne comprenait pas encore. Mais, Placeat de concordia, avait dit Pie IX. Et, faisant taire toutes ses appréhensions, M. Caval obéissait; et il se portait garant de la soumission de ses fils. Sachons reconnaître la grandeur de son geste.

L'expérience allait prouver que ses craintes étaient mal fondées. En attendant, il accélérait la solution finale. La Propagande pouvait parler: elle savait qu'elle serait entendue.

$\mathrm{La}$ grande et belle décision du 22 décembre 1865 était promise au succès. Mais sur le moment, elle apparut trop à Montréal comme une rupture avec un passé jusqu'ici intangible, pour être acceptée d'emblée. De là, recours devant les tribunaux civils, appels à Rome, controverses dans les journaux, inquiétude et malaise dans la ville. Mais c'est là une autre histoire qu'il n'est pas dans notre propos de raconter ici.

LÉON POULIOT, S.J.

Nous nous excusons, encore une fois, auprès de nos collaborateurs, du retard que, par force majeure, nous sommes obligés de consentir à la publication de leurs articles. Ils peuvent être assurés qu'on leur fera place aussitôt que possible dans nos prochaines livraisons. Plusieurs de ces travaux sont déjà composés par notre imprimeur.

N.D.R. 